\title{
Validación del cuestionario Maslach Burnout Inventory-Student Survey (MBI- SS) en contexto académico colombiano
}

\author{
Validation of Maslach Burnout Inventory-Student Survey \\ (MBI-SS) in Colombian academic context
}

\author{
Christian Hederich-Martínez ${ }^{1}$, Carmen Cecilia Caballero-Domínguez ${ }^{2}$ \\ 'Universidad Pedagógica Nacional, ${ }^{2}$ Universidad del Magdalena, Colombia
}

Forma de citar: Hederich-Martínez, C., E Caballero-Domínguez, C. (2016). Validación del cuestionario Maslach Burnout Inventory-Student Survey (MBI-SS) en contexto académico colombiano. Revista CES Psicología, 9(1), 1-15.

\begin{abstract}
Resumen
La evaluación del síndrome del burnout académico se ha hecho, tradicionalmente, a partir de la adaptación del MBI-General Survey (MBI-GS) al contexto académico colombiano, lo que se denomina MBIStudent Survey (MBI-SS). En ausencia de datos normativos propios, la mayoría de las investigaciones toman los datos normativos del MBI-GS para la interpretación de los resultados. El artículo examina la validez factorial del MBI-SS, la consistencia de las escalas planteadas y contrasta los resultados obtenidos en su aplicación a una muestra de 820 estudiantes de carreras del área de la salud en universidades de la costa Caribe colombiana con los planteados en la nota técnica que presenta los datos normativos. Los resultados indican un buen ajuste del instrumento a lo esperado, pero cuestionan los datos normativos utilizados para la interpretación de los resultados. Específicamente, los resultados muestran que la muestra presenta mayores niveles de agotamiento, menores niveles de cinísmo y similares niveles de autoeficacia que la muestra de trabajadores españoles. Se elaboran varias interpretaciones de los hallazgos.
\end{abstract}

Palabras clave: Burnout Académico, Instrumentos de Evaluación, Educación Superior

\begin{abstract}
The assessment of the academic burnout syndrome has been traditionally conducted by the MBI- General Survey (MBI-GS) in an academic context, which is called as the MBI-Student Survey (MBI-SS). Due to the lack of appropriate normative data, in order to interpret the results most of researches take the normative data from the MBI-GS. This article examines the factorial validity of MBI-SS findings, the reliability of the considered scales and compares the obtained results after conducting a survey to 820 students enrolled in the academic health field at universities in the Caribbean coast of Colombia, with the outline based on the technical mark that presents the normative data. The results indicate an efficient adjustment of the instrument, but question the normative data employed in order to carry out the interpretation of the results. Specifically, the results show a sample with higher levels of exhaustion, lower levels of cynicism and similar levels of self-efficacy than the sample of Spanish workers. Some interpretations of the results are discussed.
\end{abstract}

Keywords: Academic Burnout, Occupational Stress, Evaluation Instruments, Higher Education.

DOI: http://dx.doi.org/10.21615/cesp.9.1.1

${ }^{1}$ Doctor en Psicología, Profesor titular de la Universidad Pedagógica Nacional. Coordinador e investigador del Grupo de Investigación Estilos Cognitivos. hederich@pedagogica.edu.co

${ }^{2}$ Doctora en Psicología. Docente de la Universidad del Magdalena. Investigadora del Grupo de Investigación Psicología y Salud.ccaballero@unimagdalena.edu.co

Esta obra está bajo una licencia de Creative Commons Reconocimiento-NoComercial-SinObraDerivada 4.0 Internacional 


\section{Introducción}

El síndrome del burnout académico es una condición de estrés prolongado e insidioso que se deriva de condiciones del individuo y del contexto organizacional de la institución universitaria. Esta condición parece estar relacionada con que la institución no facilita el desempeño académico del estudiante al no proveer suficientes recursos, a la existencia de dinámicas negativas para la realización del trabajo académico y a excesivas exigencias en el mismo, entre otras (Caballero, Hederich, \& Palacio, 2012).

En los años setenta, Freuderberger (1974) se refirió al burnout como condición de los profesionales de la salud, definida como una combinación de cansancio emocional crónico, fatiga física, pérdida de interés por la actividad laboral, baja realización personal y deshumanización en el cuidado y atención a los pacientes. Maslach (1976) definió el síndrome como un estado de estrés crónico producido por el contacto de los profesionales de la salud con los pacientes, que lleva a la extenuación del profesional y a un distanciamiento emocional con sus pacientes, junto con dudas acerca la capacidad para realizar efectivamente su labor.

Para 1978 se extendió el estudio del burnout hacia otras profesiones. Aunque no existe una definición unánimemente aceptada sobre esta versión del síndrome (Garden, 1987), a lo largo de los años se ha establecido un consenso en el sentido de que se trata de una respuesta al estrés laboral caracterizada por actitudes y sentimientos negativos hacia el trabajo y hacia el propio rol profesional, así como por una vivencia de encontrarse emocionalmente agotado (Maslach $\varepsilon$ Jackson, 1981). Más recientemente,
Schaufeli y Enzmann (1998) lo han definido como un estado mental persistente y negativo, relacionado con el trabajo en individuos normales, que se caracteriza principalmente por agotamiento emocional, acompañado de distrés, un sentimiento de reducida competencia, poca motivación y el desarrollo de actitudes disfuncionales en el trabajo.

La extensión del síndrome al ámbito académico, surge de la presunción de que los estudiantes universitarios, al igual que cualquier profesional, se encuentran con presiones y sobrecargas propias de la labor académica (Garcés de los Fayos, 1995). Bresó (2008) señala que los estudiantes, al igual que cualquier trabajador, mantienen una relación de compensación directa e indirecta con la institución universitaria, evidenciada en apoyos económicos, becas, reconocimientos $\mathrm{O}$ premios. Esta presunción permite investigar las respuestas del individuo ante la tensión y sus implicaciones en su sensación de bienestar frente a los estudios (Bresó, 2008: Caballero, Abello, \& Palacio, 2007). De allí que, al igual que los trabajadores formales, los estudiantes universitarios pueden llegar a encontrarse en condiciones riesgosas caracterizadas por demandas concurrentes, excesivas e inapropiadas, crónicamente irresueltas, o resueltas de forma inadecuada, que conllevan, con el tiempo, a daños psicosociales importantes (Bresó, Caballero, E González, 2015). Así, el burnout académico es definido como una condición del estudiante caracterizada por la sensación de no poder dar más de sí mismo, una actitud de crítica negativa, desvalorización, pérdida del interés, de la trascendencia y del valor de los estudios y dudas crecientes acerca de la propia capacidad para realizarlos (Schaufeli, Martínez, Marques Pinto, Salanova, $\varepsilon$ Bakker, 2002). 
Instrumentos para evaluación del burnout académico

El inventario MBI (Maslach Burnout Inventory) de Maslach y Jackson (1981) ha sido el instrumento más utilizado por la comunidad investigativa para la evaluación de síndrome del burnout en contextos asistenciales $y$ ha sido validado en diferentes contextos culturales y laborales (Carlotto \& Câmara, 2006; Martínez, 2010; Olivares-Faúndez, Mena-Miranda, MacíaSepulveda, \& Jélvez-Wilke, 2014; Rostami, Abedi, Schaufeli, Ahmadi, E Sadeghi, 2014). También conocido como MBI-Human Services Survey (MBI-HSS), inicialmente fue utilizado en el ámbito de la salud y evalúa, mediante 22 ítems, las tres variables centrales que caracterizan al burnout en los profesionales de la salud: agotamiento, despersonalización y baja eficacia profesional. El agotamiento hace referencia a la sensación de no poder dar más de sí mismo; la despersonalización, por su parte, hace alusión a una actitud fría y a un trato distante hacia los pacientes y, por último, la baja eficacia profesional, se refiere a la sensación de no hacer adecuadamente las tareas y ser incompetente en la solución de las dificultades del trabajo (Pérez et al., 2012).

El desarrollo de la investigación que amplió el carácter de burnout a otras profesiones, transformó el MBI-HSS en el MBI-General Survey (MBI-GS) (Schaufeli, Leiter, Maslach, E Jackson, 1996). Este instrumento es una versión abreviada del MBI-HSS y agrupa 16 ítems que podrían ser aplicados a cualquier grupo profesional. Evalúa dos de las dimensiones de la versión original: agotamiento y baja eficacia profesional, pero no hace referencia al contexto de las emociones negativas relacionadas con el sufrimiento y la demanda de los pacientes sino a las relaciones con el trabajo. Así, la escala de despersonalización, contenida en la versión original, cambió su nombre por la de "cinismo", e incluye las actitudes negativas, de indiferencia o de distancia hacia el trabajo en general (Martínez, 2010).

Para la operacionalización del burnout al contexto académico, Schaufeli, Salanova, González-Romá y Bakker (2002) adaptaron el MBI-GS a estudiantes universitarios, lo cual dio origen al cuestionario MBI-SS (Maslach Burnout Inventory-Student Survey). Para hacerlo, se partió de la presunción de que la actividad académica en el rol del estudiante es equivalente a la de un trabajador formal (Bresó, 2008).

La aplicación del MBI-SS demostró que, al igual que los trabajadores, los estudiantes pueden reflejar agotamiento por las demandas del estudio (agotamiento), además de actitudes de desinterés, autosabotaje frente a las actividades académicas y dudas acerca del valor del estudio (cinismo) y sentimiento de incompetencia como estudiantes (autoeficacia negativa) (Bresó, Llorens, E Salanova, 2005; Bresó \& Salanova, 2005; Salanova, Grau, Martínez \& Llorens, 2004; Salanova, Martínez, Bresó, Llorens, E Grau, 2005).

Schaufeli, Martínez, et al., (2002), examinaron las características psicométricas del MBI-SS en muestras de 1661 estudiantes de tres países: España, Portugal y Holanda. Los resultados mostraron, en los tres países, una estructura factorial de tres factores que se ajustaba perfectamente a la esperada (agotamiento, cinismo y autoeficacia) con buenos niveles de confiabilidad (agotamiento, entre .74 y .80 dependiendo del país; cinismo, entre .79 a .86 y autoeficacia entre .67 y .76).

En general, la estructura factorial obtenida en los diferentes estudios sobre el MBI-SS 
coincide con lo planteado en su diseño. Así lo encontraron Carlotto y Câmara (2006), quienes estandarizaron el MBI-SS en una población de 514 estudiantes brasileros. En este estudio, el análisis factorial de los ítems del instrumento de nuevo identificó los tres factores esperados: agotamiento emocional, autoeficacia y cinismo, encontrando una solución trifactorial con niveles relativamente satisfactorios de consistencia interna $(.81, .74$ y $\quad .59$ respectivamente).

En una reciente línea de investigación desarrollada por Salmela-Aro y sus colaboradores, se planteó y validó un nuevo instrumento, de nueve ítems, para la determinación de burnout académico: el School-Burnout Inventory (SBI-U) (SalmelaAro, Kiuru, Leskinen, \& Nurmi, 2009). Este instrumento fue diseñado originalmente en idioma inglés, si bien ya ha sido traducido al castellano y aplicado en muestras de población española y latinoamericana (Aguilar-Bustamante \& Riaño-Hernandez, 2013; Boada-Grau, Merino-Tejedor, Sánchez-García, Prizmic-Kuzmica, E VigilColet, 2015; Merino, Delgadillo, $\quad \varepsilon$ Caballero, 2013; Moyano \& RiañoHernández, 2013). Todos los resultados publicados hasta el momento muestran una estructura trifactorial prácticamente idéntica a la presentada por el MBI-SS, asî como niveles de fiabilidad aceptables para cada escala.

Acerca de los baremos y los datos normativos para el MBI-SS, no se conocen estudios ni propuestas específicas para este instrumento. Usualmente, para el MBI-SS se asumen los datos normativos recomendados en nota técnica NTP-732 del Instituto Nacional de Seguridad e Higiene en el Trabajo de España, que orienta la interpretación del MBI-GS. Esta nota técnica define los niveles de burnout de acuerdo a los percentiles obtenidos en una muestra de trabajadores españoles de diferentes ocupaciones, de manera que el primer cuartil agrupa los valores bajos en cada una de las dimensiones, el segundo y tercer cuartil los valores medios, y el cuarto cuartil los valores altos. Así, se considera que un individuo presenta el síndrome de burnout si muestra valores altos en agotamiento y cinismo y bajos en autoeficacia.

Esta interpretación de los resultados podría ser adecuada para los trabajadores españoles, pero no serlo para los estudiantes, y más aún de otras latitudes. Dado este vacío de información en la interpretación específica del MBI-SS, se requiere de estudios que examinen la pertinencia de los parámetros planteados en la norma técnica NTP-732 para la interpretación del instrumento en su versión académica. Así en el presente trabajo examinaremos las características psicométricas del cuestionario del Maslasch Burnout Inventory-Student Survey (MBI-SS) para la población de estudiantes universitarios del área de la salud en la ciudad de Barranquilla (Colombia) y la pertinencia y justificación de su comparación con los parámetros planteados en la nota técnica.

\section{Método}

\section{Instrumentos}

El inventario que se examinará, Maslach Burnout Inventory-Student Survey (MBI-SS), de Schaufeli, Martínez, et al.. (2002), es un cuestionario para la evaluación del síndrome de burnout académico de aplicación colectiva y diligenciamiento individual. Evalúa la sensación de no poder dar más de sí mismo, tanto física como psíquicamente (agotamiento), la presencia de una actitud negativa de desvalorización 
y pérdida del interés por el estudio (cinismo) y la existencia de dudas acerca de la propia capacidad para realizar el trabajo académico (autoeficacia académica) (Bresó, $\underline{2008)}$.

Todos los ítems de cada una de estas tres subescalas se puntúan en una escala de frecuencia de 7 puntos, que va desde 0 (nunca) a 6 (siempre). Cinco ítems evalúan agotamiento, cuatro evalúan cinismo y seis ítems evalúan autoeficacia académica, para un total de 15 reactivos.

Para el cómputo de los resultados, se toman los datos normativos recomendados en la nota técnica NTP-732 del Instituto Nacional de Seguridad e Higiene en el Trabajo de España, que orienta la interpretación del MBI-GS. Esta nota define los niveles de burnout de acuerdo a los percentiles obtenidos en una muestra de 1963 trabajadores españoles de diferentes ocupaciones, de manera que el primer cuartil agrupa los valores bajos en cada una de las dimensiones, el segundo y tercer cuartil los valores medios, y el cuarto cuartil los valores altos. En la tabla 1 se presentan los parámetros definidos en la nota técnica NTP-732.

Tabla 1. Datos normativos para la corrección de las puntuaciones de las escalas de burnout $(\mathrm{N}=1.963)$

\begin{tabular}{lcccc}
\hline & & Agotamiento & Cinismo & Eficacia profesional \\
\hline Muy bajo & $>5 \%$ & $<0.4$ & $<0.2$ & $<2.83$ \\
Bajo & $5-25 \%$ & $0.5-1.2$ & $0.3-0.5$ & $2.83-3.83$ \\
Medio (Bajo) & $25-50 \%$ & $1.3-2.0$ & $0.6-1.24$ & $3.84-4.5$ \\
Medio (Alto) & $50-75 \%$ & $2.1-2.8$ & $1.25-2.25$ & $4.51-5.16$ \\
Alto & $75-95 \%$ & $2.9-4.5$ & $2.26-4$ & $5.17-5.83$ \\
Muy Alto & $>95 \%$ & $>4.5$ & $>4.0$ & $>5.83$ \\
Media Aritmética & & 2.12 & 1.50 & 4.45 \\
Desviación Típica & & 1.23 & 1.30 & 0.90 \\
\hline
\end{tabular}

Tomado de: España, Ministerio de Trabajo y Asuntos sociales

\section{Participantes}

La muestra del estudio corresponde a 820 estudiantes de los programas de medicina, psicología, fisioterapia y enfermería de tres universidades privadas de la ciudad de Barranquilla (Colombia). En la muestra prevalece el sexo femenino $(79,1 \%)$ sobre el masculino (20,9\%). Las edades están entre los 17 y los 27 años, con una edad media de 20.24 años y una desviación típica de 2.06 años.

El estado civil soltero (96\%) predomina en los estudiantes, y, en menor porcentaje se encuentran estudiantes casados $(2,4 \%)$, en unión libre (1\%) y separados (.6\%). Respecto al estrato socioeconómico, tal y como lo define el Departamento Nacional de Estadística de Colombia (DANE), la mayoría de los estudiantes residen actualmente en viviendas que corresponden a un estrato socioeconómico identificado como 2 -o medio bajo (35\%)-, seguido del estrato 3 -bajo (23,8\%)-, el estrato 4 -medio (18\%) - y el estrato 5 medio alto (1.4\%)-

Procedimiento

Previo al proceso de aplicación, a los estudiantes se les ofreció la información básica acerca de la investigación, elemento indispensable para la firma del consentimiento informado. Posteriormente se llevó a cabo la aplicación del 
instrumento en los diferentes grupos que consintieron su participación en la investigación. La aplicación fue hecha personalmente por uno de los autores del artículo. El comité de ética de la Universidad del Norte, en Barranquilla (Colombia), avaló los aspectos éticos de la investigación.

\section{Análisis estadístico}

Para el procesamiento de los datos se usó el software estadístico IBM SPSS Statistics en su versión 20. En un primer momento, la matriz de datos fue sometida a una depuración a través del análisis de frecuencias y de puntajes extremos y estereotipados.

Completada la depuración, para dar respuesta al objetivo, se realizaron diferentes procedimientos de análisis de confiabilidad y análisis de validez factorial del instrumento. El análisis de confiabilidad se hizo mediante el cálculo del Alpha de Cronbach, con el fin de determinar la consistencia interna de cada escala, y la contribución de los diferentes reactivos a la misma. Por su parte, el análisis de validez factorial fue hecho mediante un análisis factorial exploratorio de componentes principales, para determinar la coincidencia entre la estructura subyacente del instrumento y la planteada por sus autores. Para determinar si los datos se ajustaban al análisis factorial se calcularon el índice de Kaiser-MeyerOlkin (KMO), utilizando como criterio de corte el índice $\mathrm{KMO}>0.7$ y la prueba de esfericidad de Bartlett. El análisis se calculó con rotación oblicua Oblimin con Kaiser para dimensiones correlacionadas.

Para la valoración de los resultados respecto de los obtenidos en la nota técnica NTP-732 referida a trabajadores españoles, se examinaron pruebas t para una muestra en las que se compararon las medias obtenidas en cada dimensión con los valores de las medias anotados en la nota técnica.

\section{Resultados}

Análisis de validez factorial del cuestionario MBI (SS)

A fin de examinar la coherencia entre la estructura planteada para el instrumento y los resultados empíricos se examinó un análisis factorial exploratorio, con el método de componentes principales, sobre los 15 ítems de la escala del burnout académico Maslach Burnout Inventory-Student Survey (MBI-SS), de Schaufeli, Martínez, et al. (2002) utilizando un método de rotación oblicuo (Oblimín).

Como se acostumbra en este tipo de análisis, primero debe verificarse el cumplimiento de las condiciones necesarias para la ejecución del análisis factorial, y el particular el cumplimiento de los requisitos de multicolinealidad de los datos. Esto se hace a través de dos procedimientos: la medida de adecuación muestral de $\mathrm{KMO}$, que da como resultado un índice de 869 considerado como muy bueno (Hair, Anderson, Tatham, E Black, 1999); por su parte, el contraste de esfericidad de Barlett, muestra un Chi cuadrado de 3357.84, gl $=105, \mathrm{p}<.001$. Estos dos resultados indican la pertinencia de correr un análisis factorial sobre estos 15 elementos.

Una primera inspección de los niveles de comunalidad de los 15 ítems muestra que 14 de ellos, presentan extracciones superiores a .4 , tal y como se espera, pero uno, el ítem Efi 6-35, de autoeficacia, muestra un nivel de comunalidad extremadamente bajo (.19). Ver tabla 2. 
Ahora, utilizando el criterio del autovalor mayor que 1, el análisis de componentes principales identifica tres componentes que explican un $52.75 \%$ de varianza total. Las cargas factoriales se presentan en la mitad izquierda de la tabla 2. Tal como se observa, en el primer factor se ubican todos los ítems de autoeficacia académica con una varianza explicada del 29.38\%, excepción hecha del ítem Efi6_35, que ya habíamos identificado por su baja comunalidad y que queda ubicado en el tercer factor. En el segundo factor, con una varianza de $14.19 \%$, se ubican todos los ítems de la dimensión de agotamiento y, por último, en el tercer factor, con una varianza explicada de $9.18 \%$, se encuentran todos los ítems de la escala de cinismo, más el ítem ya señalado de autoeficacia, que muestra en este factor una carga pequeña y negativa.

Tabla 2.Extracciones y matrices de estructura de los análisis factoriales sobre 15 y 14 ítems, respectivamente.

\begin{tabular}{|c|c|c|c|c|c|c|c|c|}
\hline \multirow[t]{2}{*}{ Ítems } & \multicolumn{4}{|c|}{$\begin{array}{l}15 \text { ítems } \\
\text { Componente }\end{array}$} & \multicolumn{4}{|c|}{$\begin{array}{l}14 \text { ítems } \\
\text { Componente }\end{array}$} \\
\hline & Extrac. & 1 & 2 & 3 & Extrac. & 1 & 2 & 3 \\
\hline & & & & & & - & & \\
\hline Efi_3_9 En mi opinión soy un buen estudiante & .64 & .80 & -.15 & -.38 & .64 & .80 & -.17 & -.37 \\
\hline Efi_2_7 Creo que contribuyo efectivamente con & & - & & & & - & & \\
\hline las clases en la universidad & 62 & .79 & -.21 & -.30 & .62 & .79 & -.23 & -.31 \\
\hline Efi_5_15 Durante las clases, tengo la seguridad & & - & & & & - & & \\
\hline de que soy eficaz en la finalización de las cosas & 62 & .78 & -.18 & -.25 & .62 & .78 & -.20 & -.26 \\
\hline Efi_1_5 Puedo resolver de manera eficaz los & & - & & & & - & & \\
\hline problemas relacionados con mis estudios & .55 & .73 & -.20 & -.38 & .55 & .73 & -.22 & -.38 \\
\hline Efi_4_10 Me estimula conseguir objetivos en & & - & & & & - & & \\
\hline mis estudios & .52 & .71 & -.04 & -.37 & .52 & .71 & -.06 & -.35 \\
\hline $\begin{array}{l}\text { Ago_4_22 Me encuentro agotado físicamente } \\
\text { al final de un día en la universidad }\end{array}$ & .57 & .05 & .75 & .06 & .58 & .05 & .75 & .11 \\
\hline Ago_5_25 Estoy exhausto de tanto estudiar & .60 & .15 & .74 & .34 & .60 & .16 & .74 & .39 \\
\hline $\begin{array}{l}\text { Ago_2_3 Estoy cansado en la mañana cuando } \\
\text { me levanto y tengo que afrontar otro día en la }\end{array}$ & & & & & & & & \\
\hline universidad & .54 & .20 & .73 & .13 & .54 & 20 & .73 & .19 \\
\hline $\begin{array}{l}\text { Ago_1_1 Las actividades académicas de esta } \\
\text { carrera me tienen emocionalmente "agotado" }\end{array}$ & .54 & .23 & .7 & 21 & .55 & 23 & 73 & 25 \\
\hline Ago_3_20 Estudiar o ir a clases todo el día es & & & & & & & & \\
\hline un tensión para mi & .43 & .22 & .64 & .10 & .42 & .22 & .64 & .16 \\
\hline $\begin{array}{l}\text { Cin_3_27 He perdido interés en la carrera } \\
\text { desde que empecé en la universidad }\end{array}$ & .62 & .32 & .24 & .78 & .62 & 32 & .25 & .79 \\
\hline Cin_4_33 He perdido entusiasmo por mi & & & & & & & & \\
\hline carrera & .60 & .34 & .25 & .77 & .63 & .34 & .25 & .79 \\
\hline $\begin{array}{l}\text { Cin_1_4 Me he distanciado de mis estudios } \\
\text { porque pienso que no serán realmente útiles }\end{array}$ & .50 & .33 & .18 & .70 & .51 & 33 & .18 & .72 \\
\hline $\begin{array}{l}\text { Cin_2_13 Dudo de la importancia y valor de } \\
\text { mis estudios }\end{array}$ & .40 & .29 & .15 & .63 & .43 & .29 & .15 & .65 \\
\hline Efi_6_35 He aprendido muchas cosas & & - & & & & & & \\
\hline interesantes durante mi carrera* & .19 & .19 & .11 & -.39 & & & & \\
\hline
\end{tabular}

Método de extracción: Análisis de componentes principales. Método de rotación: Normalización Oblimin con Kaiser.

Los ítems aparecen ordenados por las cargas factoriales en el análisis de 15 factores. Se señalan en negrilla las cargas factoriales mayores a 0.4 
Un análisis más detallado del contenido del ítem Efi 6-35 muestra que las dificultades pueden iniciar con su contenido. La redacción del ítem, "He aprendido muchas cosas interesantes durante mi carrera" podría no identificarlo con el concepto de autoeficacia, tal y como fue definido: "la creencia en las propias capacidades para organizar y ejecutar cursos de acción que son requeridos para manejar situaciones futuras" (Bandura, 1999, p. 20). Las dificultades podrían estar relacionadas con la redacción inicial del ítem en el instrumento MBI-GS: "He conseguido muchas cosas valiosas en este puesto", que podría ser adecuado en el contexto laboral, en tanto "conseguir cosas valiosas" es uno de los objetivos principales en un puesto de trabajo, pero su transcripción al contexto académico, hecha de la forma "He aprendido muchas cosas interesantes durante mi carrera" podría no ser la indicada. Posiblemente, una transcripción que enfatice resultados tales "he obtenido muy buenas calificaciones durante mi carrera", pueda mostrar mejores relaciones con el factor de autoeficacia.

Dadas las dificultades relacionadas con la inclusión del ítem Efi 6-35, se eliminó este ítem y se recalculó el análisis factorial. Como en el caso anterior, la verificación de los supuestos muestra la pertinencia de correr el análisis sobre los 14 elementos restantes: $\mathrm{KMO}=.87 \mathrm{y}$, para el contraste de esfericidad de Barlett, se obtiene un Chi cuadrado de 3294.52, gl= 91, p<.001. De nuevo, los ítems se discriminan en tres factores, que alcanzan a explicar el 55.8\% de la varianza. Los factores son los mismos que los obtenidos en el análisis anterior, si bien en este caso la varianza explicada es levemente mayor y algunas de las cargas factoriales aparecen levemente incrementadas. Las comunalidades de los
14 ítems son superiores a 0.43 y las cargas factoriales en cada factor aparecen en la mitad derecha de la tabla 2.

Tal y como se observa, en este segundo análisis factorial los ítems se discriminan perfectamente de acuerdo con lo esperado. En el primer factor, que explica el $31.20 \%$ de la varianza, se agrupan los cinco ítems de autoeficacia; en el segundo, que explica el 14,95\%, quedan los cinco ítems de agotamiento, y en el tercero, que explica el $9,65 \%$, los cuatro ítems de la escala de cinismo. Esta solución muestra un instrumento con una muy adecuada validez factorial y representa una fuerte evidencia acerca de la validez de constructo del cuestionario, especialmente después de excluir el ítem Efi 6-35, al menos en su presente transcripción. Lo que sigue, que solo se tomarán los resultados derivados de los 14 ítems del cuestionario.

Por último, vale la pena mencionar que las correlaciones bivariadas entre los puntajes de las dimensiones son positivas, moderadamente altas y significativas a niveles inferiores a .001. Esto se da puesto que el factor 1, de autoeficacia, quedó definido con cargas factoriales negativas, por lo que, en su sentido actual no representa autoeficacia, sino su opuesto: ineficacia académica. Así, la correlación entre cinismo e ineficacia muestra los valores más elevados $(r=.43, p<.001)$; la correlación entre agotamiento y cinismo es un poco menor $(r=.32, p<.001)$ y la correlación entre agotamiento e ineficacia muestra los menores valores $(r=.26, p<$ $.001)$.

Análisis descriptivos de las escalas

La tabla 3 presenta los estadísticos de las diferentes subescalas y de la escala total. 
Su cálculo se realizó a partir de la media de los ítems que conformaban cada una de las escalas. En la medida en que cada ítem se puntuaba entre los valores 0 y 6 , las medias de las subescala y de la escala total se unifican entre valores mínimos de 0 y máximos de 6.

Tabla 3. Estadísticos descriptivos de las subescalas y MBI-SS

\begin{tabular}{|c|c|c|c|c|c|c|c|}
\hline & & \multicolumn{2}{|c|}{ Agotamiento } & \multicolumn{2}{|c|}{ Cinismo } & \multicolumn{2}{|c|}{ Eficacia } \\
\hline \multicolumn{2}{|l|}{ Media } & \multicolumn{2}{|c|}{2.34} & \multicolumn{2}{|r|}{0.54} & \multicolumn{2}{|c|}{4.41} \\
\hline \multicolumn{2}{|c|}{ Desviación típica } & \multicolumn{2}{|c|}{1.08} & \multicolumn{2}{|r|}{0.80} & \multicolumn{2}{|c|}{0.99} \\
\hline \multicolumn{2}{|c|}{ Z de Kolmogorov-Smirnov (p) } & \multicolumn{2}{|c|}{$.09(<.001)$} & \multicolumn{2}{|c|}{$.27(<, 001)$} & \multicolumn{2}{|c|}{$.10(<.001)$} \\
\hline \multicolumn{2}{|c|}{ Alfa de Cronbach } & \multicolumn{2}{|c|}{.77} & \multicolumn{2}{|r|}{.72} & \multicolumn{2}{|c|}{.82} \\
\hline \multicolumn{2}{|c|}{ No. de ítems } & \multicolumn{2}{|r|}{5} & \multicolumn{2}{|r|}{4} & \multicolumn{2}{|c|}{5} \\
\hline \multirow{6}{*}{$\begin{array}{c}\text { Rangos } \\
\text { de } \\
\text { puntaje }\end{array}$} & Muy Bajo (>5\%) & 14 & $1.7 \%$ & 390 & $47.6 \%$ & 68 & $8.3 \%$ \\
\hline & Bajo (5\%-25\%) & 120 & $14.6 \%$ & 221 & $27.0 \%$ & 183 & $22.3 \%$ \\
\hline & Medio-bajo (25\%-50\%) & 234 & $28.5 \%$ & 47 & $5.7 \%$ & 151 & $18.4 \%$ \\
\hline & Medio-alto (50\%-75\%) & 226 & $27.6 \%$ & 124 & $15.1 \%$ & 185 & $22.6 \%$ \\
\hline & Alto (75\%-90\%) & 196 & $23.9 \%$ & 34 & $4.1 \%$ & 206 & $25.1 \%$ \\
\hline & Muy alto (>95\%) & 30 & $3.7 \%$ & 4 & $.5 \%$ & 27 & $3.3 \%$ \\
\hline
\end{tabular}

Tal y como se observa, el $27.6 \%$ de la muestra presenta niveles altos o muy altos de agotamiento. Las pruebas de normalidad de esta escala muestran que la distribución difiere de forma muy significativa de la correspondiente a la curva normal. El valor del alfa se encuentra dentro de niveles aceptables.

Por su parte la subescala de cinismo, presenta una media que alcanza, apenas, un valor de 0.54 . Solo el $4.6 \%$ de la muestra presenta una actitud alta o muy alta de indiferencia, autosabotaje y distanciamiento frente a los estudios. Las pruebas de normalidad de la escala de cinismo muestran que la distribución difiere de forma muy significativa de la distribución correspondiente a la curva normal. El valor del alfa en esta escala es aceptable.

Por su parte, la subescala de autoeficacia académica, conformada por la suma total de los valores de los cinco ítems, presenta valores bajos, o muy bajos, en el $30.6 \%$ de la muestra. Estos valores podrían ser considerados preocupantes. También en este caso, la forma de la distribución difiere de manera significativa de la curva normal. El alfa de Cronbach alcanza su valor máximo en esta escala.

Combinando los datos de las tres subescalas, es posible obtener la medida de la prevalencia del síndrome en la muestra. En total, solo 20 sujetos, que representan el $2.4 \%$ de la muestra total cumplen con todas las condiciones para tener el síndrome del burnout: niveles altos, o muy altos, de agotamiento y cinismo, y niveles bajos, o muy bajos, de autoeficacia.

Resultados y comparaciones con el estándar de la norma española

La figura 1 muestra la comparación de los resultado entre lo obtenido en el presente proyecto, y lo planteado por la nota técnica NTP-732 del Instituto Nacional de Seguridad e Higiene en el Trabajo de España, que orienta la interpretación del MBI-GS. En relación con la escala de 
agotamiento, los datos de los estudiantes colombianos $(\mathrm{M}=2.34$, DT $=1.08)$ muestran ser levemente mayores que los reportados en la nota técnica para la población de trabajadores españoles $(M=2.12, \mathrm{DT}=1.23)$. La diferencia muestra ser significativa en una prueba t para una muestra con el valor $t(819)=5.9 . p<.001, d=0.22,95 \% \mathrm{CI}[.14, .29]$.

Por su parte, la escala de cinismo es la que muestra mayores diferencias entre las dos muestras, en tanto los estudiantes colombianos muestran niveles muy bajos de cinismo $(M=0.54, \mathrm{DT}=0.80)$, bastante alejados de los encontrados en trabajadores españoles ( $M=1.5 . \mathrm{dt}=1.30)$. La diferencia es ampliamente significativa en una prueba t de una muestra $t(819)=-34.32$, $p<.001, d=-0.96,95 \%$ CI $[-1.01,-0.90]$.

Por último, es interesante anotar que prácticamente no aparecen diferencias en la prueba de autoeficacia entre la muestra de estudiantes colombianos ( $M=4.41 \mathrm{DT}=0.99)$ y la de trabajadores españoles $(\mathrm{M}=4.45$, $\mathrm{DT}=0.90)$. Correspondientemente, la diferencia entre la media obtenida por los estudiantes colombianos y el estándar de los trabajadores españoles no muestra ser significativa en la prueba $t$ para una muestra $t(819)=-1.02, p=.309, d=-.03,95 \% \mathrm{Cl}$ [-0.1. 0.03].

Figura 1. Comparación de las medias obtenidas por estudiantes colombianos y las reportadas para trabajadores españoles para las subescalas del MBI-SS.

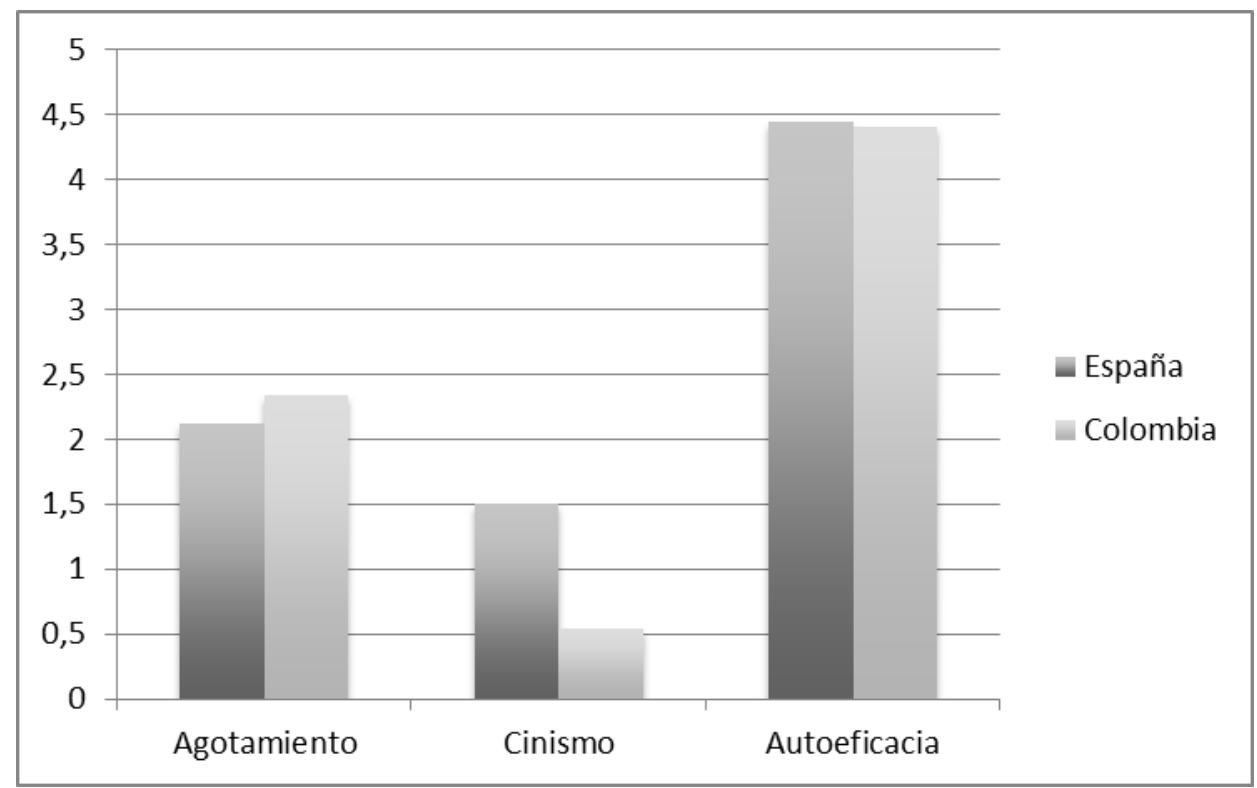

Fuente: Datos propios (Colombia) y nota técnica NTP-732 del Instituto Nacional de Seguridad e Higiene en el Trabajo de España.

\section{Discusión}

Dos grandes objetivos tenía este estudio: establecer las características psicométricas del cuestionario del Burnout Inventory-Student Survey (MBI-SS) de Schaufeli, Martínez, et al. (2002) para la población universitaria del área de la salud en Barranquilla (Colombia) y verificar la pertinencia de los parámetros planteados en la norma técnica NTP-732 para la interpretación de los resultados derivados de la aplicación de este instrumento. 
Al respecto del primer objetivo planteado, el análisis de ítems permitió identificar una inconsistencia estadística en la escala de ineficacia académica que sugirió la necesidad de eliminar uno de los ítems: "He aprendido muchas cosas interesantes durante mi carrera académica". A partir de esta modificación se favoreció una mejor consistencia de la subescala de autoeficacia académica. Eliminado este ítem, los resultados muestran que el MBI-SS se comportó de manera satisfactoria y consistente con otros estudios realizados de validación y análisis de características psicométricas del mismo, lo que indica su adecuación para la identificación y medición del burnout académico en la población de estudiantes universitarios en el contexto de la costa caribe colombiana. Las subescalas del MBI-SS mostraron un alto grado de consistencia interna. Estos resultados son similares a los obtenidos en los estudios de validación de Schaufeli. Martínez, et al., (2002), Carlotto y Câmara, (2006), y Rostami, et al. (2014).

Respecto del segundo objetivo planteado, la verificación de la pertinencia de los parámetros planteados en la norma técnica NTP-732 para la interpretación de los resultados, debe señalarse que nuestros resultados son consistentes con los encontrados por Tomaschewski-Barlem et al. (2014). En este estudio, los autores aplicaron MBI-SS en una muestra de 168 estudiantes de pregrado de enfermería en una universidad pública del sur de Brasil. Aunque los resultados no se presentan en rangos, se anota que la muestra tiene altos índices de agotamiento, indicados por una media de 4.0 en los ítems de la dimensión, y niveles bajos de cinismo (1.80) y altos de autoeficacia (4.54). En general, no se registra ni un solo caso que cumpla las tres condiciones, y que por lo tanto pueda ser propiamente de burnout, en la muestra.
Un punto que resulta muy interesante de estos resultados es el hallazgo de las similitudes y diferencias entre las muestras de estudiantes colombianos del presente estudio y las muestras de trabajadores españoles utilizadas para la estandarización del MBI-GS, de donde se obtuvieron los parámetros para la interpretación del MBISS. Los resultados indicaron que los estudiantes colombianos muestran mucho mayores índices de agotamiento y mucho menores niveles de cinismo que los trabajadores españoles; en los niveles de autoeficacia no se encontraron diferencias. Al respecto surge la pregunta acerca del origen de estas diferencias.

Varias hipótesis, no excluyentes sino más bien con efectos concomitantes, pueden ser planteadas para explicar estas diferencias. La primera, relevaría las diferencias presentes entre la situación académica y la situación laboral. La segunda, estaría indicando diferencias culturales entre las dos muestras. La tercera hipótesis indicaría diferencia en las etapas evolutivas en las que están los individuos de las diferentes muestras.

$\mathrm{Al}$ respecto de las diferencias entre la situación académica y la laboral, debe anotarse que a pesar de las similitudes entre el mundo laboral y el estudiantil, es presumible que algunas particularidades de estos espacios puedan explicar las diferencias obtenidas. En particular, los mayores niveles de agotamiento de los estudiantes podrían ser explicados por la naturaleza de la actividad académica, que no da descanso al final del día, e incluso al final de la semana, a diferencia de la actividad laboral que tiene un horario más claro y, al menos en los niveles operativos, no supone tareas adicionales para llevar a casa. En este sentido, es comprensible que los estudiantes puedan encontrarse más agotados que los trabajadores, sobre todo 
en los períodos de finalización del semestre en los que se acumulan las obligaciones y exámenes (Galbraith, E Merrill, 2012).

En cuanto a las diferencias en los niveles de cinismo, estas también podrían ser explicadas por la naturaleza de los dos tipos de actividad. En la situación laboral, el trabajador establece una relación de dependencia con su salario, que compromete sus niveles de vida y su supervivencia y la de su familia. En una situación de estrés laboral extremo, la gran mayoría de los trabajadores no pueden dejar de trabajar, aunque así lo quisieran; solo tienen la posibilidad de cambiar de trabajo, lo cual depende del estado del mercado laboral. En esta situación, el trabajador desarrollará, con mayor probabilidad, una actitud disfuncional hacia el trabajo caracterizada por el desinterés, pérdida del valor y distrés; esto es, una actitud de cinismo. La situación de los estudiantes es diferente, en tanto el abandono de sus estudios no compromete, usualmente, su estabilidad económica ni la de su familia, al menos en el corto plazo. En ese sentido, el estudiante que encuentra insatisfactoria su actividad puede abandonarla, sin que este abandono tenga consecuencias mayores. Así, aunque no tenemos antecedentes que lo apoyen en la literatura, las más bajas tasas de cinismo podrían ser explicables por una deserción temprana de aquellos estudiantes que encontraron excesivamente estresante su actividad académica.

En relación con la segunda hipótesis, que indicaría que las diferencias entre las dos poblaciones puedan estar relacionadas con diferencias de tipo cultural es poco lo que se puede avanzar. La muestra de estudiantes colombianos fue obtenida íntegramente de universidades localizadas en la ciudad de Barranquilla, localizada en el litoral Caribe colombiano. La población mayoritariamente asentada en esta zona ha sido descrita ampliamente en estudios de la antropología cultural como con una tendencia al relajamiento, al goce, la fiesta $\mathrm{y}$ al disfrute del momento presente (Gutiérrez de Pineda, 2000). Es posible que esto pueda explicar las diferencias en cinismo, mucho menores en esta muestra, pero no es muy claro cómo puede hacerlo con los niveles de agotamiento encontrados. De cualquier forma, la influencia de este tipo de contextos de naturaleza cultural es una hipótesis compleja que no puede ser descartada y que no debe ser reducida a características puntuales del grupo cultural.

La tercera hipótesis que podría explicar las diferencias son las características de las etapas evolutivas en las que se encuentran los individuos de las dos muestras. La muestra de trabajadores está constituida por adultos. La muestra de estudiantes está conformada por adolescentes o adultos jóvenes. En esta última etapa, se sabe que la importancia del grupo social tiene su valor máximo en la identificación propia y la estructuración de su personalidad; es una etapa caracterizada por la presencia permanente y constante de interacciones sociales con el grupo de referencia. En este sentido, el grupo y la interacción social permea toda la actividad, facilitando que el estrés pueda ser afrontado con una red de apoyo social, lo cual podría actuar como factor protector hacia el desarrollo de actitudes negativas hacia el estudio, y explicar así el menor cinismo encontrado en la muestra de estudiantes.

Como se observa, las hipótesis planteadas pueden contribuir a la explicación de las diferencias encontradas. Es factible que la explicación completa de las diferencias encontradas deba ser atribuida a una combinación de las hipótesis desarrolladas. Esto pone en cuestionamiento el uso de los parámetros obtenidos del MBI-GS para la interpretación de los resultados del MBISS. Aparentemente, se requiere desarrollar 
nuevos parámetros para la interpretación de este instrumento en la versión académica de manera que se describa de mejor manera el estrés asociado con este tipo de actividad.

Por otro lado, es relevante mencionar que el presente estudio, aunque examina una muestra de estudiantes colombianos de buen tamaño, ésta se limita a estudiantes del área de ciencias de la salud en tres universidades localizadas en una ciudad de la costa caribe colombiana. Por las posibles influencias de aspectos culturales en las dimensiones examinadas, ello puede limitar la validez externa de los resultados presentados. Futuros trabajos con muestras de universitarios en otras áreas y otras ciudades colombianas y latinoamericanas permitira superar esta limitación.

\section{Referencias}

Aguilar-Bustamante, M., \& Riaño-Hernandez, D. (2013). Propiedades psicométricas del "School Burnout Inventory" SBI en población colombiana adolescente [Psychometric properties of the "School Burnout Inventory" SBI in teenage Colombian population]. Paper presented at the 34th Interamerican Congress of Psychology, 15-19 July, Brasilia (Brazil). [link]

Bandura, A. (1999). Ejercicio de la eficacia personal y colectiva en sociedades cambiantes. En Bandura, A., Autoeficacia: cómo afrontamos los cambios de la sociedad actual (pp. 19-54). Madrid: Descleé de Brower. [link]

Bresó, E (2008). Well-being and Performance in Academic Settings: The Predicting Role of Selfefficacy. [Disertación doctoral no publicada]. Universidad Jaume I de Castellón, Castellón, España. [in press]

Bresó, E., Caballero, C., \& González, O. (2015). Burnout en estudiantes universitarios. Psicología desde el Caribe, 32(3). (En prensa). [link]

Bresó, E., Llorens, S., E Salanova, M. (2005). Creencias de eficacia académica y engagement en estudiantes universitarios. Jornades de Foment de la Investigació. |Recuperado de www.uji.es/bin/publ/edicions/jfi9/psi/6.pdf].

Bresó, E., E Salanova, M., (2005). Efectos significativos del uso de las creencias de ineficácia como componente del burnout acadêmico en estudiantes universitários. Jornades de Foment de la Investigació. Recuperado de www.uji.es/bin/publ/edicions/jfi8/psi/32.pdf

Boada-Grau, J., Merino-Tejedor, E., Sánchez-García, J. C., Prizmic-Kuzmica, A. J., E Vigil-Colet, A. (2015). Adaptation and psychometric properties of the SBI-U scale for academic burnout in university students. Anales de Psicología, 31(1), 290-297. doi: http://dx.doi.org/10.6018/analesps.31.1168581

Caballero, C. (2012). Prevalencia y factores asociales al síndrome de burnout académico. [Disertación doctoral no publicada]. [in press]. 
Caballero, C., Hederich, C., E Palacio, J. (2010). El burnout académico. Delimitación del síndrome y factores asociados con su aparición. Revista latinoamericana de Psicología, 42(1), 131-146. [link]

Caballero, C., Abello, R., E Palacio, J. (2007). Relación del burnout y rendimiento académico con la satisfacción frente a los estudios. Avances en Psicología Latinoamericana, 25(2), 98-111. [link]

Carlotto, M. S., E Câmara, S. G. (2006). Características psicométricas do Maslach Burnout InventoryStudent Suvey (MBI-SS) em estudantes universitarios brasileiros. Psico-USF, 11(2), 167-173. [link]

España, Instituto Nacional de Seguridad e Higiene en el Trabajo (200?) NTP-732: Síndrome de estar quemado por el trabajo "Burnout" (III): Instrumento de medición. En: http://www.insht.es/InshtWeb/Contenidos/Documentacion/FichasTecnicas/NTP/Ficheros/701a750/ ntp 732.pdf

Freudenberger, H. J. (1974). Staff burnout. Journal of Social Issues, 30(1), 159-165. [link]

Garcés de los Fayos, E. (1995). Burnout en niños y adolescentes: Un nuevo síndrome en psicopatología infantil. Psicothema, 7(1), 33-40. [link]

Garden, A. (1987). Depersonalization: A valid dimension of burnout? Human Relations, 40(9), 545560. link!

Galbraith, C., E Merrill, G. (2012). Academic and Work-Related Burnout: A Longitudinal Study of Working Undergraduate University Business Students. Journal of College Student Development, 53(3), 453-463. doi:10.1353/csd.2012.0044

Gutiérrez de Pineda, V. (2000). Familia y Cultura en Colombia. Tipologías. Funciones y Dinámica de la Familia. Manifestaciones múltiples a través del mosaico cultural y sus estructuras sociales. Medellín: Universidad de Antioquia.

Hair, J., Anderson, R., Tatham, R., E Black, W. (1999). Análisis Multivariante. Prentice Hall.

Martínez, A. (2010). El síndrome de burnout. Evolución conceptual y estado actual de la cuestión. Vivat Academia, 112, 1-40. En http://www.ucm.es/info/vivataca/numeros/n112/DATOSS.htm

Maslach, C. (1976). Burned-out. Human Behavior, 9(5), 16-22. [link]

Maslach, C., E Jackson, S.E. (1981). The measurement of experienced burnout. Journal of Occupational Behaviour, 2, 99-113. [link]

Merino, C., Delgadillo, A., E Caballero, R. (2013). ¿Burnout en adolescentes?: Validez estructural del inventario de burnout escolar (SBI)]. Paper presented at the 34th In-teramerican Congress of Psychology, 15-19 July, Brasilia (Brazil). [in press]

Moyano, N., E Riaño-Hernández, D. (2013). Burnout escolar en adolescentes españoles: Adaptación y validación del School Burnout Inventory. Ansiedad y Estrés, 19, 95-103. [link]

Olivares-Faúndez, V., Mena-Miranda, L., Macía-Sepulveda, F., \& Jélvez-Wilke, C. (2014). Validez factorial del Maslach Burnout Inventory Human Services (MBI-HSS) en profesionales chilenos. Universitas Psychologica, 13(1), 145-159. doi:10.11144/Javeriana.UPSY13-1.vfmb [link] 
Pérez, C., Parra P., Fasce, E., Ortiz, L., Bastías, N., E Bustamante C. (2012). Estructura Factorial y Confiabilidad del Inventario de Burnout de Maslach en Universitarios Chilenos. Revista Argentina de Clínica Psicológica, 21(3), 255-263. [link]

Rostami, Z., Abedi, M., Schaufeli, W., Ahmadi, S., E Sadeghi A. (2014). The Psychometric Characteristics of Maslach Burnout Inventory Student Survey: Among Students of Isfahan University. Zahedan Journal of Research in Medical Sciences, 16 (9), 55-58. [link]

Salanova, M. Grau, R., Martínez, I., \& Llorens, S. (2004). Facilitadores, Obstáculos, rendimientos académicos relacionados con la satisfacción con los asuntos del estudio. Periódico El País Universal.

Salanova, M., Bresó, E., E Shaufeli, W. (2005). Hacia un modelo espiral de las creencias de eficacia en el estudio de burnout y del engagement. Revista Ansiedad y Estrés, 11(2-3), 215-231. [link]

Salanova, M., Martínez, I., Bresó, E., Llorens, S., E Grau, R. (2005). Bienestar psicológico en estudiantes universitarios: facilitadores y obstaculizadores del desempeño académico. Anales de Psicología, 21(1), 170-180. [link

Salmela-Aro, K. (2012). Gendered pathways in school burnout among adolescents. Journal of Adolescence, 31, 89-95. [link]

Salmela-Aro, K., Kiuru, N., Leskinen, E., E Nurmi, J. E. (2009). School-Burnout Inventory (SBI) Reliability and validity. European Journal of Psychological Assessment, 25, 48-57. [link]

Schaufeli, W., E Ezmann, D. (1998). The burnout companion to study and practice: A critical analysis. London: Taylor \& Francis.

Schaufeli, W.B., Leiter, M.P., Maslach, C., E Jackson, S.E. (1996). The Maslach Burnout InventoryGeneral Survey. In C. Maslach, S.E. Jackson, \& M.P. Leiter (Eds.), Maslach Burnout Inventory. Palo Alto, CA: Consulting Psychologists Press

Schaufeli, W., Martínez, I., Marques Pinto, A., Salanova, M., E Bakker, A. (2002). Burnout and engagement in university students: A cross-national study. Journal of Cross - Cultural Psychology, 33(5), 464-481. [link]

Schaufeli, W. B., Salanova, M., González-Romá, V., \& Bakker, A. (2002). The measurement of burnout and engagement: A confirmatory factor analytic approach. Journal of Happiness Studies, 3, 71-92. [link]

Tomaschewski-Barlem, J.G., Lunardi, V.L., Lunardi, G.L., Barlem, E.L.D., Silveira, R.S., E Vidal, D.A.S. (2014). Burnout syndrome among undergraduate nursing students at a public university Rev. Latino-Americana de Enfermagem, 22(6), 934-941. doi: 10.1590/0104-1169.3254.2498

Recibido: Agosto 26-2015 Revisado: Febrero 3-2016 Aceptado: Marzo 18-2016 